\title{
LAS VELARES SORDAS LATINAS Y EN ESPECIAL EL USO DE LA $K$ EN LOS GRAMÁTICOS LATINOS
}

M." José López de Ayala y Genovés

$U N E D$

El trabajo que dedico a los gramáticos latinos se comprende en el contexto de una amplia investigación sobre los efectos de la persistencia del grafema $K$.

No es mi intención exponer los «efectos», ni siquiera su "persistencia», sino más bien presentar por primera vez, conjuntamente, cuál era el pensamiento de estos estudiosos, desde Varrón hasta San Isidoro, acerca de las velares sordas latinas y en especial de la letra $K$ sobre la que San Isidoro en sus Originum libri seu Etymologiae dice: $K$ litteram Saluius ludimagister pri/m]us Latinis adiecit, ut in sono discrimen faceret duarum litterarum $C$ et $Q$ y a la que como a los veintitrés signos del alfabeto latino afecta la definición dada por Prisciano en Institutiones grammaticae ${ }^{2}$ : Littera est pars minima uocis compositae, expresada por todos los gramáticos en general, como encontramos en Diomedes ${ }^{3}$, Pseudo Probo y Donato 5 . Todo esto es completado con la aportación de Sergio en De syllabis, de pedibus, de accentibus, de distinctione ${ }^{6}$ : dixit partem minimam esse litteram uocis articulatae, quod, cum omnis oratio soluatur in uerba, uerba denuo soluantur in syllabas, rursum syllabae soluantur in litteras y matizado por Carisio en su

ISID. Orig. $1,4,12$.

PRISC. Gramm. 1,3 = GL II 6,6.

DIOM. $2=$ GL I 421,15 .

- [PROB. Inst. $=$ GL IV 48,33.

'DON. Gramm. 1 = GL IV 367,9.

${ }^{6}$ SERG. = GL IV 475,6. 
Ars Grammatica?: littera est elementum uocis articulatae, elementum est uniuscuiusque rei initium, a quo sumitur incrementum et in quod resoluitur.

Los signos del alfabeto latino, exceptuando dos de origen griego, son latinos y se agrupan en vocales y consonantes; éstas al ser estudiadas por los gramáticos, en especial las consonantes mudas, se expresan de la siguiente manera, como escribe Pseudo Probo en Instituta artium ${ }^{8}$ mutae consonantium litterae sunt numero nouem.hae nec per se proferuntur nec per se syllabam facere possunt. per se hae non proferuntur, siquidem uocalibus litteris subiectis sic nomina sua definiunt, ut puta be ce de ge ha ka pe qu te, per se autem syllabam facere non possunt.

No todos los gramáticos, como señala Lambert ${ }^{9}$, tratan con la misma profundidad el tema de litteris y, como consecuencia, lo referente a las velares. Este punto, debido a la existencia de tres signos o grafias diferentes y a su evolución, ha tenido un trato especial por parte de todos; unos para abusar de la $k$ en posición inicial, en especial si se encontraba la vocal $a$, como Probo y Máximo Victorino y otros, como Arusiano Mesio, para no facilitar en su obra ningún nombre que empiece con $\mathrm{ca}$ o $\mathrm{cl}$, e incluso admitir su colocación dentro del orden alfabético entre la $i$ y la $l$. También encontramos ejemplos en Velio Longo que, aceptando la grafia con $k$, critica a quienes utilizan en sus cartas la fórmula karissime.

Donato, evitando emitir juicios sobre otros, opina que es propio de ignorantes el seguir la costumbre de escribir siempre con $k$, y finalmente Diomedes, Servio, Sergio, Pompeyo, Cledonio, Terenciano Mauro y Prisciano, dejan reducido su uso a un número muy limitado de palabras, a las que ellos llamarán notae, es decir, signo de abreviatura de palabras muy concretas y en usos determinados. En este estudio de las velares sordas latinas $\mathrm{y}$, en especial del uso de la $k$, los gramáticos siguen también, en una gran mayoría, el uso tradicional y son pocos los que sobre este tema han formado una doctrina concreta, aunque sí puede deducirse de su estudio y confrontación una serie de principios más o menos determinados, que a continuación vamos a ir detallando.

En el presente estado de cosas, el más antiguo en el tiempo de estos gramáticos es Varrón, del que conocemos su obra fragmentariamente, revelándose en ella más como sabio que como pedagogo; sus sucesores inmediatos llegan a nosotros sólo de nombre, hasta que aparece la figura de Quintiliano, que redacta todo un plan de gramática latina, seguido por autores posteriores, en el que trata algunas cuestiones delicadas o de controversia.

'CHAR. 1, 3, pág. 4,10 B.

[PROB.] Inst. = GL IV 50,5.

- Lambert, Ch., La Grammaire latine selon les Grammariens latines du IV et du V siècle. Paris, 1908. 
Palemón, Probo, Cominiano y Plinio el Viejo, sólo subsisten con extractos de su obra en algunos casos insignificantes. Terenciano Mauro, Terencio Escauro y Velio Longo, que viven en el siglo I-II d. C., se ocupan de cuestiones de ortografia, y pueden ayudar en algunas cuestiones restringidas.

Para encontrar los verdaderos gramáticos, es decir, los estudiosos que brindan por escrito sus enseñanzas de un modo metódico, tenemos que llegar al siglo IV d. C. en las figuras de Carisio, Diomedes y, sobre todo, Donato. No menos importante que ellos fue Prisciano, siglos v-vI, completando esta panorámica la figura de San Isidoro (VI-VII).

El conjunto de gramáticos de los siglos IV-VI se bifurca en dos direcciones: los llamados tradicionalistas, entre los que situaríamos a Mario Plocio Sacerdote, Carisio, Diomedes y Dositeo, y los considerados doctrinarios, es decir, Donato (comentado por Servio), Sergio, Cledonio, Pompeyo, Focas y el erudito Prisciano, que, conocedor de la literatura griega, concilia la teoría con los hechos.

Estos gramáticos afrontan el tema de las velares latinas de muy distinta manera, lo cual permite elaborar una distribución de los mismos según la cuestión que planteen.

I. Así, un primer grupo considera a las tres velares sordas $c, k, q$, conjuntamente. Ya Terenciano Mauro en De litteris, de syllabis, de metris ${ }^{10}$ se expresa según sigue: $k$ perspicuum est littera quod uacare possit. De modo parecido se recoge en los Commenta Einsidlensia ${ }^{11}: k$ et $q$ nihil aliud habent quam c; afirmación esta expresada, más tarde, por Prisciano en su obra Institutiones grammatica ${ }^{12}$ : quamuis in uaria figura et uario nomine sint $k$ et $q$ et c, tamen,... pro una litera accipi debent, completándola con otro pasaje de la misma obra ${ }^{13}: k$ enim et $q$, quamuis figura et nomine uideantur aliquam habere differentiam, cum $c$ tamen eandem tam in sono uocum quam in metro potestatem continent. et $k$ quidem penitus superuacua est: nulla enim uidetur ratio, cur a sequente haec scribi debeat: "Carthago» enim et "caput", siue per $c$ siue per $k$ scribantur, nullam faciunt nec in sono nec in potestate eiusdem consonantis differentiam, donde expone que no hay ninguna razón para escribir $k$ en lugar de $c$, pues la velar $k$ se recoge en latín con la grafia $c$, y no se puede olvidar que el grupo de $k u$ permaneció en latín ante vocal distinta de $u$ y que su grafia es $q u$; por lo tanto el apéndice desaparece ante vocal $u$ (secutus/sequor) y ante consonante (coctus/coquo).

10 TER.-MAVR. = GL VI 331,204.

" COMM.EINSID. = GL Suppl. 225,8.

12 PRISC. Gramm. 1,17 = GL II 13,14.

13 ID. Ib. $1,14=$ GL II 12,5 . 
Pero otros gramáticos encuentran elementos de diferenciación, siquiera sea fonética o grafemática. Tal Mario Victorino en Ars grammatica ${ }^{14}$ : nam muta et otiosa parte, qua $c$ incipit, pro qualitate coniunctae sibi uocis supremum exprimit sonum, non nihil tamen interest, utra earum prior sit, $c$ seu $q$ siue $k$. quarum utramque exprimi faucibus, alteram distento, alteram producto rictu manifestum est, en donde deja bien claro cuál es el modo de articulación, que de la misma manera es ratificado por Marciano Capela en su obra De nuptiis Philologiae et Mercurii ${ }^{15}$ : $K$ faucibus palatoque formatur.

En efecto, un concepto muy analizado por los gramáticos, con relación a las tres velares sordas $c, k, q$, es la definición de mutae superuacuae, que nos lleva a fijarnos en lo expresado por Pseudo Probo en sus Instituta artium $^{16}$ y situarlo en relación con el proceso de reflexión de los gramáticos posteriores así: Mutae consonantium litterae sunt numero nouem... per se hae non proferuntur, siquidem uocalibus litteris subiectis sic nomina sua definiunt, ut puta be ce de ge ha ka pe qu te. per se autem syllabam facere non possunt, scilicet quoniam mutae litterae, si misceantur, sonum syllabae facere non reperiuntur, ut puta bc dg $t k p q$ et cetera talia. nunc et in his mutis superuacue quibusdam $k$ et $q$ litterae positae esse uidentur quod dicant c litteram earundem locum posse complere, ut puta Carthago pro Karthago. nunc hoc uitium etsi ferendum puto, attamen pro quam quis est qui sustineat cuam? et ideo non recte hae litterae quibusdam superuacue constitutae esse uidentur. Esta afirmación viene ratificada por Marciano Capela en su obra De nuptiis Philologiae et Mercurii ${ }^{17}$ : si enim $H$ aspirationi dabitur, superuacuaeque erunt $Q$ et $K$.

En general, no es necesaria la velar $k$ para aquellas palabras que se pronuncian igual, es decir, lo que los gramáticos expresan con la fórmula c littera possit implere, que queda recogida en bastantes ocasiones, así Mario Victorino en Ars grammaticae ${ }^{18}$ : mutae... sunt autem numero nouem... ex his superuacuae quibusdam uidentur $k$ et $q$, quia $c$ littera harum locum possit implere; y en otro lugar ${ }^{19}$ : pro $k$ et $q$ c littera facillime haberetur; este autor recoge en otros pasajes lo siguiente, asi ${ }^{20}:$ nam nostri eruditi uno ore $h$ quidem et $k$ superuacuas esse dixerunt ${ }^{21}: k$ et $q$ superuacue numero litterarum inseri doctorum plerique contendunt, scilicet quod c littera harum officium possit implere, finalmente ${ }^{22}:$ quae... superuacua uidentur? $k$ et $q . .$. quia c littera ha-

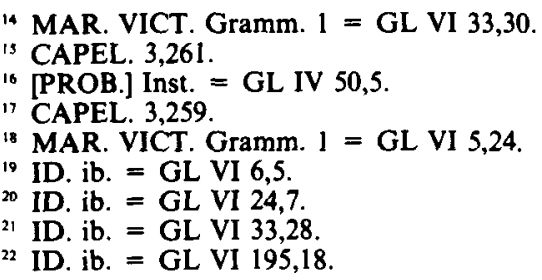


rum locum possit explere; y todo este conjunto es ratificado por Donato ${ }^{23}$ y Dositeo ${ }^{24}$ en su obra respectiva Ars grammatica y más tarde por Carisio en Ars grammatica ${ }^{25}$ : mutae... sunt autem numero nouem... ex his superuacuae quibusdam uidetur $k$ et $q$, quod c littera horum locum possit inplere, y en la misma línea continúa Diomedes en Ars grammatica ${ }^{26}$, que viene a ser completado por lo expresado en Explanationes in Donati Artem $^{27}$ : non enim sunt necessariae... $k$ et $q$ ideo, quod $c$ littera harum locum possit implere. Finalmente, Cledonio en su Ars grammatica ${ }^{28}$, da por hecha esta realidad al escribir: duae superuacuae $k$ et $q$, quia harum locum $c$ implet; y esta misma expresión possit explere queda subrayada por Audax en su obra De Scauri et Palladii libris excerpta ${ }^{29}$. A partir de este momento, aparece el término superflua utilizado por Sergio en De syllaba, de pedibus, de accentibus, de distinctione $^{30}$, y recogido por Jerónimo en su Interpretatio nominum Hebraicorum $^{31}$ : de $k$ superfluum est mentionem facere, cum etiam apud Latinos exceptis Kalendis superflua iudicetur, afirmado por Pompeyo en su Commentum artis Donati ${ }^{32}$ y de manera tajante $\mathrm{en}^{33}$ tolle $k$ et $q$, quas superfluas uocant.

II. Como ha podido observarse, una apertura a la diferenciación está presente en la idea de los autores que parecen desentenderse personalmente de la identidad, quibusdam uidentur, y variantes (Pseudo Probo, Capela, Mario Victorino, Carisio).

Pero ello sería muy poco; lo cierto es que la diferenciación llega a ser postulada claramente, especialmente con criterios distribucionales.

Aparece como significativo que los gramáticos, al precisar la vocal que para ellos era propia de la pronunciación de la velar $k$, puedan utilizar expresiones en algunos casos muy diferenciadas.

1. Esto permite resaltar que hay gramáticos para quienes el nombre de la $K$ nunca termina en $e$, así Servio en su Commentarius in Donati artem ${ }^{34}$

DON. Gramm. 1 = GL IV 368,7.

24 DOSITH. $=$ GL VII 382,6 .

29 CHAR. 1,3,p.5,22 B.

26 DIOM. $2=$ GL I 423,10.

EXPLAN. = GL IV 520,22.

CLED. = GL V 28,15.

AVD. = GL VII 326,19.

30 SERG. = GL IV 477,14.

" HIER. Nom. Hebr.,p.28,28 L.

32 POMP. GR. = GL V 110,4 .

3 ID. ib. = GL V 111,7.

34 SERV. Comm. = GL IV 422,34. 
Pero otros gramáticos encuentran elementos de diferenciación, siquiera sea fonética o grafemática. Tal Mario Victorino en Ars grammatica ${ }^{14}$ : nam muta et otiosa parte, qua c incipit, pro qualitate coniunctae sibi uocis supremum exprimit sonum, non nihil tamen interest, utra earum prior sit, $c$ seu $q$ siue $k$. quarum utramque exprimi faucibus, alteram distento, alteram producto rictu manifestum est, en donde deja bien claro cuál es el modo de articulación, que de la misma manera es ratificado por Marciano Capela en su obra De nuptiis Philologiae et Mercurii ${ }^{15}$ : $K$ faucibus palatoque formatur.

En efecto, un concepto muy analizado por los gramáticos, con relación a las tres velares sordas $c, k, q$, es la definición de mutae superuacuae, que nos lleva a fijarnos en lo expresado por Pseudo Probo en sus Instituta artium $^{16}$ y situarlo en relación con el proceso de reflexión de los gramáticos posteriores asi: Mutae consonantium litterae sunt numero nouem... per se hae non proferuntur, siquidem uocalibus litteris subiectis sic nomina sua definiunt, ut puta be ce de ge ha ka pe qu te. per se autem syllabam facere non possunt, scilicet quoniam mutae litterae, si misceantur, sonum syllabae facere non reperiuntur, ut puta bc dg $t k p q$ et cetera talia. nunc et in his mutis superuacue quibusdam $k$ et $q$ litterae positae esse uidentur quod dicant $c$ litteram earundem locum posse complere, ut puta Carthago pro Karthago. nunc hoc uitium etsi ferendum puto, attamen pro quam quis est qui sustineat cuam? et ideo non recte hae litterae quibusdam superuacue constitutae esse uidentur. Esta afirmación viene ratificada por Marciano Capela en su obra De nuptiis Philologiae et Mercurii ${ }^{17}$ : si enim $H$ aspirationi dabitur, superuacuaeque erunt $Q$ et $K$.

En general, no es necesaria la velar $k$ para aquellas palabras que se pronuncian igual, es decir, lo que los gramáticos expresan con la fórmula c littera possit implere, que queda recogida en bastantes ocasiones, así Mario Victorino en Ars grammaticae ${ }^{18}$ : mutae... sunt autem numero nouem... ex his superuacuae quibusdam uidentur $k$ et $q$, quia c littera harum locum possit implere; y en otro lugar ${ }^{19}$ : pro $k$ et $q$ c littera facillime haberetur; este autor recoge en otros pasajes lo siguiente, asi ${ }^{20}$ : nam nostri eruditi uno ore $h$ quidem et $k$ superuacuas esse dixerunt ${ }^{21}: k$ et $q$ superuacue numero litterarum inser $i$ doctorum plerique contendunt, scilicet quod c littera harum officium possit implere, finalmente ${ }^{22}:$ quae... superuacua uidentur? $k$ et $q . .$. quia c littera ha-

14 MAR. VICT. Gramm. $1=$ GL VI 33,30.

is CAPEL. 3,261.

16 [PROB.] Inst. = GL IV 50,5.

17 CAPEL. 3,259.

18 MAR. VICT. Gramm. $1=$ GL VI 5,24.

19 ID. ib. = GL VI 6,5.

20 ID. ib. $=$ GL VI $24,7$.

${ }^{21}$ ID. ib. = GL. VI 33,28.

${ }_{22}$ ID. ib. = GL VI 195,18 . 
rum locum possit explere; y todo este conjunto es ratificado por Donato ${ }^{23}$ y Dositeo ${ }^{24}$ en su obra respectiva Ars grammatica y más tarde por Carisio en Ars grammatica ${ }^{25}$ : mutae... sunt autem numero nouem... ex his superuacuae quibusdam uidetur $k$ et $q$, quod $c$ littera horum locum possit inplere, y en la misma linea continúa Diomedes en Ars grammatica ${ }^{26}$, que viene a ser completado por lo expresado en Explanationes in Donati Artem ${ }^{27}$ : non enim sunt necessariae... $k$ et $q$ ideo, quod c littera harum locum possit implere. Finalmente, Cledonio en su Ars grammatica ${ }^{28}$, da por hecha esta realidad al escribir: duae superuacuae $k$ et $q$, quia harum locum $c$ implet; y esta misma expresión possit explere queda subrayada por Audax en su obra De Scauri et Palladii libris excerpta ${ }^{29}$. A partir de este momento, aparece el término superflua utilizado por Sergio en De syllaba, de pedibus, de accentibus, de distinctione $e^{30}$ y recogido por Jerónimo en su Interpretatio nominum Hebraicorum $^{31}$ : de $k$ superfluum est mentionem facere, cum etiam apud Latinos exceptis Kalendis superflua iudicetur, afirmado por Pompeyo en su Commentum artis Donat ${ }^{32}$ y de manera tajante $\mathrm{en}^{33}$ tolle $k$ et $q$, quas superfluas uocant.

II. Como ha podido observarse, una apertura a la diferenciación está presente en la idea de los autores que parecen desentenderse personalmente de la identidad, quibusdam uidentur, y variantes (Pseudo Probo, Capela, Mario Victorino, Carisio).

Pero ello sería muy poco; lo cierto es que la diferenciación llega a ser postulada claramente, especialmente con criterios distribucionales.

Aparece como significativo que los gramáticos, al precisar la vocal que para ellos era propia de la pronunciación de la velar $k$, puedan utilizar expresiones en algunos casos muy diferenciadas.

1. Esto permite resaltar que hay gramáticos para quienes el nombre de la $K$ nunca termina en $e$, asi Servio en su Commentarius in Donati artem ${ }^{34}$

23 DON. Gramm. 1 = GL IV 368,7.

24 DOSITH. $=$ GL VII $382,6$.

${ }^{25}$ CHAR. 1,3,p.5,22 B.

26 DIOM. $2=$ GL I $423,10$.

${ }^{27}$ EXPLAN. = GL IV 520,22.

28 CLED. = GL V 28,15.

29 AVD. = GL VII 326,19.

3) SERG. = GL IV 477,14 .

" HIER. Nom. Hebr.,p. 28,28 L.

32 POMP. GR. = GL V 110,4.

" ID. ib. = GL V 111,7.

34 SERV. Comm. = GL IV 422,34. 
dice: ex quibus tres, quoniam non desinunt in $e$, contumeliam patiuntur, $h k$ $q$, y Pompeyo en su Commentum artis Donati ${ }^{35}$ añade; sed sunt aliquae litterae, quae neque ab e inchoant neque in e desinunt, hae litterae calumnia patiuntur...k $k$ et $q$.

Paralelamente, y con formulación positiva, hay gramáticos que indican como una particularidad la presencia de la vocal $a$ en la pronunciación de ésta u otras consonantes, así en Ars Anonyma Bernensis ${ }^{36}$ : In a tria monosyllaba Latina indeclinabilia sunt, ut a ka ha; Corpus Glossariorum Latinarum $^{37}$ : kappa: ka y Ausonio en Technopaegnion ${ }^{38}$ : haec tribus in Latio tantum addita nominibus $k$, que este autor reduce a kapita, kalendae y kalumnia, aunque después veremos que ésta es una interpretación restringida.

Como se ve, se trata meramente de los nombres de estas letras; cierto que los tales tienen que ver con su distribución. Pero hasta aquí no se pasaría de un procedimiento indirecto.

2. Otros gramáticos diferencian las tres velares sordas en el sentido de que omiten la $k$, como queda recogido en los testimonios aportados por Mario Victorino en su Ars grammatica ${ }^{39}$ : Nigidius Figulus in commentariis suis nec $k$ posuit nec $q$ nec $x$; Quintiliano en De institutione oratoria ${ }^{40}$ añade: nam $k$ quidem in nullis uerbis utendum puto... quod quidam eam, quotiens a sequatur, necessariam credunt, y finalmente Prisciano en sus Institutiones grammaticae ${ }^{41}$ : auctoritas quoque tam Varronis quam Macri teste Censorino nec $k$ nec $q$ nec $h$ in numero adhibet literarum.

3. Resulta interesante, por cuanto se parece a una postura vista en 1 . referente al nombre de la letra, pero aquí llevado ya a su papel distribucional, ver cómo un gran número de gramáticos exigen que se escriba $k$ ante $a$, aunque también en esta afirmación podemos formular algunas precisiones:

A) Los hay que exigen su presencia "siempre que» se encuentre ante la vocal $a$; están representados por Mario Victorino en Ars grammatica ${ }^{42}$; uerum has quoque necessarias orthographiae ratio efficit. nam quotiens a se-

is POMP. GR. = GL V 101,18.

${ }^{36}$ ARS BERN. = GL Suppl.,p.97,29.

${ }^{37}$ CGL III 524,4.

38 AVS. Techn. 13,20.

39 MAR.-VICT. Gramm. 1 = GL VI 8, 16

* QVINT. Inst. 1, 7, 10.

" PRISC. Gramm. 1, 16 = GL II 13,8.

42 MAR.-VICT. Gamm. = GL VI 195, 20. 
quitur, per $k$ scribendum est, ut kanna, kalendae, kaput, y Velio Longo en De orthographia ${ }^{43}$ : at qui illam esse litteram defendunt, necessariam putant esse nominibus quae cum a sequente hanc litteram inchoant.

Donato en su Ars grammatica ${ }^{44}$ también escribe: qui nesciunt, quotiens a sequitur, $k$ litteram praeponendam esse non $c$, recogido también por Carisio en Ars grammatica ${ }^{45}$ : praeponitur autem $k$ quotiens a sequitur, ut kalendae, Karthago, que queda confirmado en Pompeyo en su Commentum artis Donat ${ }^{46}$ : legimus enim in litteris quia $k$ non praeponitur nisi sequente $a$; y en Cledonio, autor de un Ars grammatica ${ }^{47}$, cuando escribe: apud ueteres haec erat orthographia, ut, quotiens a sequeretur, $k$ esset praeposita, ut kaput, Kalendae.

Como se ve, no es lo mismo que tras $k$ no pueda haber sino a (Pompeyo), que la exigencia de que, ante $a$, siempre deba ir $k$ (Donato, Cledonio, si bien éste lo refiere ya sólo a los antiguos ante la evidencia, seguramente, de que en sus tiempos ya no era eso).

Pero esta afirmación de nuevo es explicitada por Audax, autor de un De Scauri et Palladii libris excerpta ${ }^{48}$ : uerum has quoque necessarias ortographiae ratio efficit. nam quotiens a sequitur, per $k$ scribendum est, ut kano kalendae kaput.

B) De posturas como la vista en Pompeyo, y siempre a base de ejemplos válidos en la historia, deriva la de algunos gramáticos que exigen que se escriba $k$ ante $a$, pero reducen el campo de algunas palabras, así aquellas de que da noticia Velio Longo en su obra De orthographia ${ }^{49}$ al decir: undem etiam religiosi quidam epistulis suscribunt karissimi per $k$ et a. quod si ideo necessaria uidetur, uerendum est ne et alias litteras requiramus, quibus aut Cicero scribatur aut commodus, que se completa con lo aportado por Terenciano Mauro en De litteris, de syllabis, de metris ${ }^{50}: k$ similiter otiosa ceteris sermonibus, tunc in usu est, cum kalendas adnotamus aut kaput: saepe Kaesones notabant hac uetusti littera, interesa por la precisión que hace de la palabra Kaesones.

El número de ejemplos es bastante reducido e incluso así expresado en Mario Sergio en su obra De syllaba, de pedibus, de distinctione ${ }^{51}: K$ uero et $q$, quae non nullis siperfluae uidentur, hanc habent rationem, ut $k$ tunc prae-

${ }^{43} \mathrm{VEL} .=$ GL VII 53, 12.

4 DON. Gramm. 1= GL IV 368, 7.

45 CHAR. 1, 3, P. 5, 26 B.

46 POMP. GR. = GL V 239, 24.

4 CLED. = GL V 28, 5 .

48 AVD. = GL VII 326, 20.

49 VEL. = GL VII 53, 13.

क) TER.-MAVR. = GL VI 349, 797.

sI SERG. = GL IV 477, 14. 
ponatur, quando a sequitur. sed hoc in paucis nominibus obseruatur, ut Kalendae Karthago.

Diomedes, en su obra Ars grammatica ${ }^{52}$, puntualiza: sed inuenimus in Kalendis et in quibusdam similibus nominibus, quod $k$ necessario scribitur, que contrasta con lo que en otra parte de la misma obra ${ }^{53}$ se escribe: $k$ consonans muta superuacua, qua utimur, quando a correpta sequitur, ut Kalendae kaput kalumniae.

En la misma línea - pero reclamando la diferencia de épocas- parecidamente a lo visto en Cledonio, Servio avisa en Commentarius in Donati artem $^{54}: k$ uero et $q$ aliter nos utimur, aliter usi sunt maiores nostri, namque illis, quotienscumque a sequebatur, $k$ praeponebant in omni parte orationis, $u t$ kaput et similia; nos uero non usurpamus $k$ litteram nisi in Kalendarum nomine scribendo; analiza el uso de esta consonante, que, aunque sitúa in omni parte orationis, reduce su grafia sólo ejemplificando la palabra Kalendae, lo que es recogido por los Fragmenta Bobiensia de littera, de accentibus, de propriis nominibus, de nomine ${ }^{5 s}$, cuando dice: $k$ nisi in paucis iam non utimur, ut in kalendis.

C) La necesidad de escribir $k$ ante $a$ en algunas palabras, también la expresa San Isidoro en su otra Originum libri seu Etymologia ${ }^{56}$ refiriéndose $^{2}$ a lemas ya mencionados y especialmente en palabras de origen griego, asi: K litteram antiqui praeponebant quotiens $A$ sequebatur, ut "kuput", "kanna», "kalamus». Nunc autem "Karthago" et "kalendae» per eandem tantum scribuntur. Omnia autem Graeca nomina qualicumque sequente uocali per $K$ sunt scribenda, y en otro lugar ${ }^{57}$ aporta una serie de nombres griegos así: Katholicus, uniuersalis, Graecum enim est. Karus Graecum nomen est, sicut est caritas, unde et caristia. Es el único que habla de los graeca nomina, sobre los que la tradición latina ha hecho un buen acopio, ampliando de manera abundante el uso de los mismos, como se puede ver por los testimonios epigráficos y por los mismos autores latinos. Así, a los aportados por San Isidoro, podemos añadir nomina et cognomina graecana, escritos en grafia latina e igualmente nombres comunes. Se recogen también en este conjunto los nombres procedentes de la $\mathrm{X}$ griega, desarrollada en latín en el grupo $k h$, así: kharis, kharitus, etc.

32 DIOM. 2 = GL I 423, 12.

33 ID. ib. = GL I 424, 29.

4 SERV. Comm. = GL IV 422, 35 .

ss FRG. LITT. = GL VII 539, 5.

56 ISID. Orig. 1, 27, 13.

"ID. ib, 10, 153. 
D) Por último, no descontando sino añadiendo, ejemplifica Arusiano Mesio, cual si de la distribución arcaica se tratase (ante $a$ y consonante), en sus Exempla elocutionum ${ }^{58}$ : kaue... kareo... kaptus... khao... kassus... klaudus... kalleo... kalco... kausatus... klam, sólo que contando ya entre ellas la $h$.

III. Atendiendo a las posturas que acaban de verse o insinuarse (brevedad de la $a$ en Diomedes), y a las referentes a situación en la palabra, el conjunto «distribucional» de $k$ en los gramáticos latinos puede clasificarse según sigue:

1.A) Entre los que la sitúan en principio de sílaba y ante $a$, citamos a Prisciano en su obra Institutiones grammaticae ${ }^{59}$ cuando afirma: dicimus praepositiua elementa tam in consonantibus quam in uocalibus... et $q$ et $k$ semper initio syllabarum ponuntur, matizando que dice concretamente en comienzo de sílaba, los que completa Pseudo Probo en De catholicis Probi ${ }^{60}$ : ideo quoniam $k$ non scribitur nisi ante a litteram puram in principio nominum uel cuiuslibet partis orationis, cum sequentis syllabae consonans principium sit, sicut docui in libro primo, que completa en otro lugar de la misma obra ${ }^{61}$ al facilitar algunos ejemplos como: Kamenae, kaleo. Este autor presenta una expresión sólo utilizada por él, es decir, litteram puram que según recoge Forcellini ${ }^{62}$ querría indicar: pura littera apud Grammaticos est ea, quae post se habet uocalem. Incert. art. Grammat. fragm. 136, p. 96, Endl. Nomina quae ante ES productam, et puram habent I litteram. Queda sin contemplar, ya pues, la posibilidad de $k$ ante consonante.

Los ejemplos aportados por Pseudo Probo, son ampliados por Mario Plocio Sacerdote en Ars grammatica ${ }^{63}$ al señalar: Kamenae Kalendae Kaleo Kareo, et siqua talia.

B) Esta afirmación da pie a que otros gramáticos puedan afirmar que la velar $k$ no puede estar en final de "palabra» como recoge Pseudo Probo en su obra De catholicis Probit ${ }^{64}$, confirmado por Mario Plocio Sacerdote en su Ars grammatica ${ }^{65}$ cuando escribe: hac littera nullum nomen terminatur,

s8 ARVS. = GL VII 488,21.

59 PRISC. Gramm. 17,7= GL III 111,20.

so [PROB.] Cath. $1=$ GL IV 10,23.

61 [ID.] ib. $3=$ GL IV 39,4.

62 Forcellini, A., Totius Latinitatis Lexicon. T.IV. Padua, 1868, p.1011.

${ }^{63}$ SACERD. 2 = GL VI 491,10.

[PROB.] Cath. $1=$ GL IV 8,19.

os SACERD. $2=$ GL VI 473,17. 
prohibición después extendida por Prisciano en sus Institutiones grammaticae $^{66}$ de manera más categórica, a saber: $k$ nullius syllabae potest esse terminalis, añadiéndole el concepto de sílaba.

2. Si todos los ejemplos hasta ahora aducidos permiten ampliar el campo de presencia de la velar $k$, no podemos perder de vista que la vocal que recoge el mayor número de frecuencias es la $a$, y que ésta precisamente para algún gramático siempre es breve. Al pasaje de Diomedes ya visto, cabe añadir a Mario Victorino en su obra Ars grammatica ${ }^{67}$, cuando dice: $K$ autem dicitur monophonos, quia nulli uocali iungitur nisi soli a breui, et hoc ita ut ab ea pars orationis incipiat; aliter autem non recte scribitur. Esta precisión queda confirmada en la mayoría de los ejemplos recogidos por los gramáticos, aunque encontramos también entre ellos dos palabras con $a$ larga, a saber, kanus y karus.

Ante la insistencia de los gramáticos de escribir con velar $k$-siempre o en algunas palabras- cuando se encuentre la vocal $a$, y esto ampliado mediante los testimonios epigráficos a cualquier lugar de la palabra, vemos que este enfoque distribucional afecta también a la posibilidad de combinarse velares de la misma grafia o diferente con una vocal para la formación de sílabas, según indica Pseudo Probo en su obra Instituta artium $^{68}$ : at uero mutae geminatae, si uocalibus occurrant nec syllabam nec sonum scilicet facere possint. quis enim $b c d k p q t \mathrm{~g}$ geminatas uocalibus misceat et sonum syllabae potest audire? et ideo hac praelatione seminocales mutas rite uidentur antecedere.

Frente a quienes exigen vocal para su pronunciación, aportamos el testimonio de Quinto Terencio Escauro, donde se expresa que la vocal puede ser reemplazada y la misma velar sorda $k$ encerrar una sílaba; así lo recoge en Artis excerpta de orthographia ${ }^{69}$ : hac tamen antiqui in conexione syllabarum ibi tantum utebantur, ubi a littera subiungenda erat, quoniam multis uocalibus instantibus, quotiens id uerbum scribendum erat, in quo retinere hae litterae nomen suum possent, singulae pro syllaba scribebantur, tamquam satis eam ipso nomine explerent, ut puta... cera, $c$ simplex et ra...ita et quotiens kanus et karus scribendum erat, quia singulis litteris primae syllabae notabantur, $k$ prima ponebatur, quae suo nomime a continebat, quia si c posuissent, cenus et cerus futurum erat, non canus et carus, de donde se deriva en los diccionarios la presencia de knus y krus.

\footnotetext{
PRISC. Gramm. 2,9= GL II 49,1.

67 MAR. VICT. Gramm. $1=$ GL VI 7,7.

${ }^{68}$ [PROB.] Inst. = GL IV 50,26.

कs SCAVR.-TER. Orth. = GL VII 14,15.
} 
IV. Algunos gramáticos también señalan una evolución en la ortografia de esta letra y en el uso hecho de la misma, distinguiendo a los maiores o antiqui del nunc o modo. Esto ya se ha visto en el testimonio de Cledonio, en su referencia a los ueteres, cuando habla de la ortografia, y esta aportación es ampliada por Quinto Terencio Escauro, autor de un Artis excerpta de orthografia ${ }^{70}$, cuando dice: ego autem contenderim magis superuacuam esse $c$ quam $k$, quoniam $k$, ut apud Graecos, satis uim etiam c litterae exprimat, sed quosdam figura deceptos, qua non solum apud nos uerum etiam apud antiquos Graecorum g littera notabatur... item XII tabulae, ubi est NI PACVNT per hanc forman, quod male quidam per c enuntiant..., credidisse nobis $k$ deesse et hanc quidem $k$ dixisse, ceterum pro ea nota adiecta a Spurio Caruilio nouam formam $G$ litterae positam. nam non fuerunt tam inperiti antiqui, ut $k$ seruarent, si aliam litteram idem sonantem habuissent. quis enim uel hebetis animi sonorum similitudinem sentire non possit?, en donde se manifiesta a favor del uso de la misma; recurriendo incluso a los testimonios de los griegos, se apoya en la existencia de los $\operatorname{signos} x$ y $\chi$, los cuales no aparecieron en un principio en el alfabeto latino, y él mismo, de una manera teorizante o con afán de emular a su predecesor, expresa en la misma obra ${ }^{71}: k$ omnibus litteris quibus $c$ et praeici et subiungi potest.

Esta interpretación es también expuesta por Pompeyo en su Commentum artis Donati ${ }^{72}$, al escribir: nihil interest, utrum per $k$ scribas an per $q$ and per c. nihil interest, tamen est aliqua differentia. apud maiores nostros longe aliae regulae fuerunt, apud nos longe aliae sunt. maiores nostri, quotienscumque a sequebatur, per $k$ scribebant: puta Kanna Karus Kalamus... quoniam a sequebatur. modo non scribuntur nisi duo admodum uerba, Karthago et Kalendae, et, ut dicit ille, quod falsum est, caput. modo enim per c scribitur; antiqui per $k$ scribebant, quando uolebant significari quod a sequebatur. stultissimam rem dicit Iuba de hac re: dicit "mihi uidetur illius rei causa scribi per $k$, ideo sic fuisse conpositum, ut, quando sententiam a iudicibus accipiebant, per $k$ scriberent caput, quasi condemnatio capitalis, quod hoc ipsum caput per $k$ condemnaretur". hanc dixit ille rationem. ergo modo nos per c scribimus.

V. Hasta este momento, hemos visto la postura de los gramáticos ante las velares sordas, en especial la $k$, y si para algunos tenía su razón de ser y otros la consideraban vacía de contenido, existe un grupo que añade un nuevo uso de la misma, a saber, el concepto de nota o abreviatura con la que se referían a algunas palabras muy concretas y en número no muy

${ }^{\circ}$ JD. ib. $=$ GL VII $15,7$.

$"$ ID. ib. = GL VII 17,18 .

72 POMP. GR. = GL V 110,5 . 
elevado. Quintiliano en De institutione oratoria ${ }^{73}$ dice: an rursus aliae redundent, praeter illam "notam» adspirationis, quae si necessaria est, etiam contrariam sibi poscit, et $k$, quae et ipsa quorundam nominum nota est, y amplía en ${ }^{74}$ : nam $k$ quidem in nullis uerbis utendum puto nisi quae significat ita, ut sola ponatur. hoc eo non omisi, quod quidam eam, quotiens a sequatur, necessariam credunt, cum sit c littera, quae ad omnis uocalis uim suam perferat, tema recogido por Velio Longo en De orthographia ${ }^{75}$ : Hinc supersunt ex mutis $k$ et $c$ et $q$, de quibus quaeritur an scribentibus sint necessariae. et qui $k$ expellunt, notam dicunt esse magis quam litteram, qua significamus kalumniam kaput kalendas: hac eadem nomen Kaeso notatur. non magis igitur in numero litterarum esse oportere quam illam notam, qua centuria, et [qua] C conuersum, quo Gaia significatur: quod notae genus iudemus in monumentis. Todo esto viene a ser ratificado con el mismo número de palabras por Quinto Terencio Escauro en Artis excerpta de orthographia ${ }^{76}$ : sed retenta est, ut quidam putant, quoniam notas quasdam significarent, ut Kaesonem et kaput et kalumnian et kalendas.

Tanto Dosíteo ${ }^{77}$, como Carisio ${ }^{78}$, en sus respectivas Artes Grammaticae, ejemplifican del mismo modo, mientras que Diomedes, en su Ars Grammatica ${ }^{79}$, se expresa de la siguiente manera: $k$ littera notae tantum causa ponitur, cum calumniam aut clades aut Caesonem quaqua aut caput significat. Finalmente, recogemos el sentir de Marciano Capela, autor de un De nuptiis Philologiae et Mercurit ${ }^{80}: k$ uero nunc nota putatur esse, nunc littera; nam eius effectus $c$ integrare non dubium est absque his: kapita kalendae kalumniae.

Del estudio realizado, afirmamos que no todos los gramáticos tratan con la misma profundidad el tema de litteris y lo referente a las velares sordas, aunque por la existencia de tres signos o grafias diferentes y su evolución, haya tenido un trato especial por parte de todos, sin olvidar que en este estudio de las velares sordas latinas, y en especial del uso de la $k$, los gramáticos siguen el uso tradicional, y son pocos los que sobre este tema han formado una doctrina concreta, aunque sí puede deducirse, de su estudio y confrontación, una serie de principios más o menos determinados.

El conjunto de principios, más o menos determinados, nosotros lo presentamos de la siguiente manera, conscientes de la diferente categoría y

${ }^{3}$ QVINT. Inst. 1,4,9.

" ID. ib. $1,7,10$.

7 VEL. = GL VII 53,5.

"SCAVR.-TER. Orth. = GL VII 14,13.

$n$ DOSITH. $=$ GL VII 385,8.

7 CHAR. 1,10, p. 7,18 B.

7 DIOM. 2 = GL I 424,27.

* CAPEL. 3,253. 
aportación de cada uno de los nombres a los que nos vamos a referir a través de las conclusiones obtenidas de su estudio.

Los gramáticos latinos afrontan el tema de las velares latinas de la siguiente manera:

I.A) Consideran las tres velares sordas conjuntamente: Terenciano Mauro, los Commenta Einsidlensia ( $k$ et $q$ nihil aliud habent quam $c$ ) y Prisciano, de quienes se concluye que no existe razón para escribir $k$ en lugar de $c$, pues la velar $k$ se recoge en latín con la grafia $c$, y no se puede olvidar que el grupo $k u$ permaneció en latín ante vocal distinta de $u$ y que su grafía es $q u$; por lo tanto, el apéndice desaparece ante vocal $u$ y ante consonante.

B) Consideran las tres velares diferenciadas, basándose en elementos:

a) Fonéticos: Mario Victorino y Marciano Capela ( $k$ faucibus palatoque formatur).

b) Grafemáticos (mutae superuacuae): Pseudo Probo, Marciano Capela. En general, no es necesaria la velar $k$ para aquellas palabras que se pronuncian igual, lo que los gramáticos expresan con la fórmula: c littera possit implere, que queda recogida en: Mario Victorino, Donato y Dositeo - ratifican al anterior-, Carisio, Diomedes, las Explanationes in Donati Artem, Cledonio y Audax.

A partir de este momento, aparece el término superflua, utilizado por: Sergio, San Jerónimo y Pompeyo (tolle $k$ et $q$, quas superfluas uocant).

Vemos, por tanto, que está presente una apertura a la diferenciación en la idea de los autores que parecen desentenderse personalmente de la identidad, quibusdam uidetur y variantes (Pseudo Probo, Marciano Capela, Mario Victorino y Carisio).

II. Diferencian las velares $(c, k, q)$ criterios distribucionales, siendo esto lo cierto y sin perder de vista que resulta muy significativo ver cómo los gramáticos, al precisar la vocal, que para ellos era propia de la pronunciación de la velar $k$, pueden utilizar expresiones en algunos casos muy diferenciadas.

De lo analizado, presentamos:

1.a) gramáticos para quienes el nombre de la $k$ nunca termina en $e$, a saber: Servio y Pompeyo. 
b) gramáticos que consideran una particularidad la presencia de $a$ en la pronunciación de esta u otras consonantes, es decir, lo recogido de la obra: Ars Anonyma Bernensis, la colección Corpus Glossariorum Latinorum y el gramático Ausonio. De aquí deducimos que se trata de las letras $a, k$ ( $k a$ ), $h(h a)$ y aunque ciertamente tienen que ver con su distribución, sólo desde un procedimiento indirecto.

2. Otros gramáticos son diferenciadores de las letras velares sordas, en el sentido de que omiten la $k$, así: Mario Victorino, Quintiliano y Prisciano. En definitiva, omiten tres consonantes, a saber: $k, q, h$.

3. Relacionado con el nombre de la letra, pero con un matiz especial llevado a su papel distribucional, un gran número de gramáticos exigen que se escriba $k$ ante $a$, asi:

a) Los que exigen su presencia, "siempre que» se encuentre ante vocal $a$. Utilizando el término neccesaria, Mario Victorino, Velio Longo, pero éste refiriéndose a aquellos que la defienden. Utilizando el término: praeponendam, Donato, Carisio, Cledonio y Pompeyo (qui $K$ non praeponitur nisi sequente a). Explicita también esta afirmación Audax.

De lo expuesto deducimos que no es lo mismo lo afirmado por Pompeyo, a saber: ante grafia $k$ siempre sigue vocal $a$, que lo manifestado por Donato y Cledonio, es decir: ante $a$ siempre debe ir $k$; pero esto mismo ellos lo reducen a los ueteres, porque en su tiempo ya no se cumplía.

b) Los que exigen que se escriba $k$ ante $a$, pero reducen el campo a "algunas palabras», así: Velio Longo, Terenciano Mauro (saepe Kaesones notabant hac uetusti littera), Mario Sergio (in paucis nominibus), Diomedes, Servio y en los Fragmenta Bobiensia.

c) En palabras de origen griego, incluso abarcando todos los nombres, sea cual sea la vocal que sigue. San Isidoro es quien hace relación a los graeca nomina.

También se recogen los nombres procedentes de la $\chi$ griega desarrollada en latín en el grupo $k h$.

d) $K$ ante $a$ y ante consonante, representada por los testimonios recogidos por Arusiano Mesio; contando entre las consonantes que siguen a $l$ y $h$.

III. Entre los que la admiten, la limitan: 
1. En principio de sílaba y ante $a$, así: Prisciano (et q et $k$ semper initio syllabarum ponuntur, referido a sílaba inicial), Pseudo Probo y Mario Plocio Sacerdote. Esta afirmación da pie a que otros gramáticos piensen que la velar $k$ no puede estar en final de "palabra».

2. La vocal $a$ recoge el mayor número de frecuencias, pero hay que tener en cuenta la cantidad. Para Diomedes, Mario Victorino y Pseudo Probo, siempre es breve, y para Quinto Terencio Escauro, Audax y Pompeyo, siempre la velar $k$ se presenta ante $a$ larga.

Finalmente cabe la posibilidad de una combinación de velares de la misma grafia o diferente, con o sin vocal para formar sílaba. Exige vocal Pseudo Probo; por el contrario, sin vocal, y la misma velar sorda encierra una sílaba, Quinto Terencio Escauro, situándonos ante los ejemplos: knus y krus.

IV. Algunos gramáticos señalan una evolución en la ortografia de esta letra y en el uso de la misma, pues distinguen entre maiores o antiqui y nunc o modo, justificando su uso en razón de la ortografia (Diomedes), por el recurso a testimonios griegos (Quinto Terencio Escauro) y por el uso indistinto de $c, k, q$, aunque se matiza, ya que puede influir en el significado de la palabra (Pompeyo), dando como ejemplo: caput y kaput (= condena).

$V$. En definitiva, la postura de los gramáticos ante las velares sordas, y en especial la velar sorda $k$, puede expresarse en términos generales de la siguiente manera:

a) para unos tiene razón de ser,

b) otros la consideran superflua y superuacua, y

c) un grupo (Quintiliano, Velio Longo, Quinto Terencio Escauro, Dosíteo y Marciano Capela), presentan un nuevo uso, a saber, el concepto de nota o abreviatura referido a un grupo no reducido de palabras.

De todo lo anteriormente expuesto con relación a la teoría de los gramáticos sobre las velares sordas, concluimos que las cuestiones planteadas se presentan según sean las velares tratadas:

a) en conjunto o diferenciadas;

b) con criterios distribucionales;

c) desde el punto de vista de las limitaciones de su uso y colocación;

d) según la variación temporal;

e) en su utilización como nota. 
A continuación presentamos dos esquemas, sobre lo tratado hasta aquí en la teoria de los gramáticos, acerca de las velares sordas $(c, k, q)$ y en especial de la $k$.

El primer esquema responde a las cuestiones planteadas, es decir, según sean tratadas las velares en conjunto o diferenciadas; con criterios distribucionales; desde el punto de vista de las limitaciones de su uso y colocación; según la variación temporal y finalmente en su utilización como nota.

El segundo esquema recoge los ejemplos aportados exclusivamente por los gramáticos latinos, por orden cronológico.

Indicamos, aunque ya lo hemos hecho anteriormente, que los gramáticos entendían la $k$ más $a$ breve, y por esta razón señalamos exclusivamente su cantidad en kanus y karus, por ser larga.

ESQUEMA SEGUN CUESTIONES PLANTEADAS

I. Velares ( $\mathrm{c} \mathrm{k}$ ) tratadas en conjunto

TER.-MAVR $=$ GL VI 331,204

COMM. EINSID. = GL Suppl. 225,8.

PRISC. Gramm. 1,17 = GL II 13,14.

$1,14=$ GL II 12,5 .

Velares (c k q) diferencidas

A) fonemáticamente:

MAR.-VICT. Gramm. $1=$ GL VI 3,30

CAPEL. 3,261.

B) grafemáticamente mutae superuacuae

[PROB.] Inst. = GL IV 50,5.

CAPEL. 3,259.

MAR.-VICT. Gramm. $1=$ GL VI 5,24.

$i b .=$ GL VI 6,5.

$\mathrm{ib.}=\mathrm{GL}$ VI 24,7 .

ib. $=$ GL VI 33,28. 
DON. Gramm. 1 = GL IV 368,7.

$$
\text { ib. }=\text { GL VI 195,18. }
$$

DOSITH. = GL VII 382,6.

CHAR. 1,3 p. 5,22 B.

DIOM. $2=$ GL I 423,10.

EXPLAN. = GL IV 520,22.

CLED. = GL V 28,15.

AVD. = GL VII 326,19.

SERG. = GL IV 477,14.

POMP, GR. = GL V 110,4.

$$
=\text { GL V } 111,7 \text {. }
$$

II. Diferenciación de las velares ( $\mathrm{c} \mathrm{q}$ ) con criterios distribucionales

1. El nombre de la $\mathrm{k}$ nunca se termina en e

SERV. Comm. = GL IV 422,34.

POMP. GR. = GL V 101,18.

- La $\mathrm{k}$ y otras consonantes presentan a en su pronunciación:

ARS BERN. = GL Suppl., p. 97,29.

CGL III 524,4 .

AVS. Techn. 13,20.

2. Prescinden absolutamente de la $\mathbf{k}$

MAR.-VICT. Gramm. 1 = GL VI 8,16.

QVINT. Inst. 1,7,10.

PRISC. Gramm. 1,16 = GL II 13,8.

3. Exigen que haya $\mathrm{k}$ :

A) 'Siempre que' se encuentre ante vocal $a$ :

MAR.-VICT. Gramm. = GL VI 195,20.

VEL. = GL VII 53,12.

DON. Gramm. $1=$ GL IV 368,7.

CHAR. 1,3, p. 5,26 B.

POMP. GR. = GL V 239,24.

CLED. = GL V 28,5. 

AVD. $=$ GL VII 326,20.

B) $k$ ante $a$, pero sólo en algunas palabras:

VEL. = GL VII 53,13.

TER.-MAVR. = GL VI 349,797.

SERG. = GL IV 477,14.

DIOM. 2 = GL I 423,12. GL I 424,29.

SERV. Comm. = GL IV 422,35.

FRG. LITT. = GL VII 539,5.

C) en palabras griegas:

ISID. Orig. 1,27,13.

10,153 .

D) $k$ ante $a$ y ante consonante:

ARVS. = GL VII 488,21.

III. Entre los que la admiten, la limitan

1.A) sólo en principio de sílaba y ante $a$ :

PRISC. Gramm. 17,7 = GL III 111,20.

[PROB.] Cath. 1 = GL IV 10,23.

GL IV 39,4 .

SACERD. 2 = GL VI 491,10.

B) $k$ no puede estar en final de palabra

[PROB.] Cath. 1 = GL IV 8,19.

SACERD. 2 = GL VI 473,17.

PRISC. Gramm. 2,9 = GL II 49,1.

2. $k$ ante $a$ breve

MAR.-VICT. Gramm. $1=$ GL VI 7,7.

DIOM. 2 = GL I 423,10.

[PROB.] Inst. = GL IV 50,26.

$k$ ante $a$ larga 
SCAVR.-TER. Orth. = GL VII 15,4.

AVD. $=$ GL VII 326,21.

POMP. GR. = GL V 110,8.

Combinación de velares de la misma grafía o diferente:

a) con una vocal:

[PROB.] Inst. = GL IV 50,26.

b) sin vocal:

SCAVR.-TER. Orth. = GL VII 14,15 .

IV. Variación temporal en su ortografia 'maiores o antiqui'

CLEDON $=$ GL V 28,5.

SCAVR. TER. Orth. = GL VII $15,7$.

GL VII 17,18 .

POMP. GR. $=$ GL V 110,5.

V. Como 'nota'

QVINT, Inst. 1,4,9.

$1,7,10$.

VEL. $=$ GL VII $53,5$.

SCAVR.-TER. Orth. = GL VII 14,13.

TER.-MAVR. = GL VI 349,799.

DOSITH. = GL VII 385,8.

CHAR. 1,10 , p. 7,18 B.

DIOM. $2=$ GL I 424,27.

CAPEL. 3. 253.

Lemas APORTADOS POR los GRAmÁticos Latinos

KAESO/KAESONES

VEL. $=$ GL VII $53,8$.

SCAVR.-TER. Orth. $=$ GL VII 14,14 .

TER.-MAVR. = GL VI 359,799.

DOSITH. = GL VII 385,9.

CHAR. 1,3, p. 7,18 B 
KALAMVS

KALCO

KALENDAE

KALENDAE

KALEO

KALLEO

KALVMNIA
POMP. GR. = GL V 110,8.

ARVS. = GL VII 488,21.

[PROB.] Inst. = GL IV 118,12 y 195,25.

VEL. = GL VII 53,7.

SCAVR.-TER. Orth. = GL VII 14,14.

TER.-MAVR. = GL VI 349,799.

SACERD. 2 = GL VI 491, 10 .

MAR.-VICT. Gramm. = GL VI 195,22.

DON. Gramm. 2 = GL IV 376,27.

COMM. EINSID. = GL Suppl. 224,34;35 y 241,8 .

DOSITH. = GL VII 385,9.

SERG. = GL IV 477,16.

CHAR. 1,3, p.5,27 y 7,18 ;

$$
\begin{aligned}
& 1,11, \text { p. } 36,3 \text {; } \\
& 1,15, \text { p. } 122,12 \text { y } \\
& 1,17, \text { p. } 153,26 \text { B. }
\end{aligned}
$$

DIOM. $1=$ GL I 328,2.

$$
2=\text { GL I 423,12 y 424,30. }
$$

SERV. Comm. = GL IV 423,1.

EXC. BOB. = GL I 549,16.

POMP. GR. = GL V 110,10.

CONSENT. GR. = GL V 348,9.

PHOC. Gramm. = GL V 428,10.

PRISC. Gramm. 18,298 = GL III 370,7-14; $18,305=$ GL III 376,14 .

ARS BERN = GL Suppl. 84,33.

CLED. = GL V 28,5.

AVD. = GL VII 326,21.

FRG. LITT. = GL VII 539,5.

[PROB.] Cath. $3=$ GL IV 39,4.

SACERD. 2 = GL VI 491,10.

ARVS. = GL VII 488,21.

VEL. = GL VII 53,6.

SCAVR.-TER. Orth. = GL VII 14,14.

DOSITH. = GL VII 385,9.

CHAR. 1,3, p.7,19 B.

DIOM. $2=$ GL I 424,30. 
KAMENAE

KANNA

KĀNVS/KANA

KAPITOLIVM

KAPTVS

KAPVT

KAREO

KARISSIMI

KARTHAGO

$\underline{\text { KĀRVS }}$

KASILINVM/ KASILINENSES

KASSVS

KAVE

KAVSATVS
[PROB.] Cath. 3 = GL IV 39,4.

SACERD. 2 = GL VI 491,10.

MAR.-VICT. Gramm. = GL VI 195,22.

SCAVR.-TER. Orth. = GL VII 15,4.

AVD. = GL VII 326,21.

POMP. GR. = GL V 110,8.

MAR.-VICT. Gramm. 1 = GL VI 23,3.

ARVS. = GL VII 488,21.

VEL. $=$ GL VII 53,7.

SCAVR.-TER. Orth. = GL VII 14,14.

TER.-MAVR. = GL VI 349,798.

MAR.-VICT. Gramm. = GL VI 195,22.

DOSITH. = GL VII 385,9.

DIOM. $2=$ GL I 424,30.

SERV. Comm. = GL IV 422,3.

CLED. = GL V 28,5.

AVD. = GL VII 326,31.

SACERD. 2 = GL VI 491,10.

ARVS. $=$ GL 488,21.

VEL. = GL VII 53,14.

[PROB.] Inst. = GL IV 50,7 Y 13.

DOSITH. = GL VII 385,10.

SERG. = GL IV 477,16.

CHAR. 1,3, p.7,19 B.

POMP. GR. = GL V 110,10.

SCAVR.-TER. Orth. = GL VII 15,4.

POMP. GR. = GL V 110,8.

MAR.-VICT. Exp. Cic. 2,57.

ARVS. = GL VII 488,21.

ARVS. $=$ GL VII 488,21.

ARVS. = GL VII 488,21. 
$\underline{\text { KHAO }}$

KLAM

KLAVDVS
ARVS. $=$ GL VII 488,21.

ARVS. = GL VII 488,21.

ARVS. = GL VII 488,21. 